\title{
BMJ Open Quality improvement in emergency obstetric referrals: qualitative study of provider perspectives in Assin North district, Ghana
}

\author{
Henrietta Afari, ${ }^{1}$ Lisa R Hirschhorn, ${ }^{1,2}$ Annie Michaelis, ${ }^{2}$ Pierre Barker, ${ }^{3}$ \\ Sodzi Sodzi-Tettey ${ }^{3,4}$
}

To cite: Afari $\mathrm{H}$,

Hirschhorn LR, Michaelis A, et al. Quality improvement in emergency obstetric referrals: qualitative study of provider perspectives in Assin North district, Ghana. BMJ Open 2014;4:e005052.

doi:10.1136/bmjopen-2014005052

- Prepublication history and additional material is available online. To view please visit the journal (http://dx.doi.org/ 10.1136/bmjopen-2014005052).

Received 13 February 2014 Revised 8 April 2014 Accepted 24 April 2014

\section{ABSTRACT}

Objective: To describe healthcare worker (HCW)identified system-based bottlenecks and the value of local engagement in designing strategies to improve referral processes related to emergency obstetric care in rural Ghana.

Design: Qualitative study using semistructured interviews of participants to obtain provider narratives. Setting: Referral systems in obstetrics in Assin North Municipal Assembly, a rural district in Ghana. This included one district hospital, six health centres and four local health posts. This work was embedded in an ongoing quality improvement project in the district addressing barriers to existing referral protocols to lessen delays.

Participants: $18 \mathrm{HCWs}$ (8 midwives, 4 community health officers, 3 medical assistants, 2 emergency room nurses, 1 doctor) at different facility levels within the district.

Results: We identified important gaps in referral processes in Assin North, with the most commonly noted including recognising danger signs, alerting receiving units, accompanying critically ill patients, documenting referral cases and giving and obtaining feedback on referred cases. Main root causes identified by providers were in four domains: (1) transportation, (2) communication, (3) clinical skills and management and (4) standards of care and monitoring, and suggested interventions that target these barriers. Mapping these challenges allowed for better understanding of next steps for developing comprehensive, evidence-based solutions to identified referral gaps within the district.

Conclusions: Providers are an important source of information on local referral delays and in the development of approaches to improvement responsive to these gaps. Better engagement of HCWs can help to identify and evaluate high-impact holistic interventions to address faulty referral systems which result in poor maternal outcomes in resource-poor settings. These perspectives need to be integrated with patient and community perspectives.

For numbered affiliations see end of article.

Correspondence to

Dr Henrietta Afari;

Henrietta_afari@hms.harvard. edu

\section{BACKGROUND}

In Ghana, maternal mortality stands at about 350 deaths/100 000 live-births, reaching higher rates in rural areas. ${ }^{1}$ Skilled-birth attendance

\section{Strengths and limitations of this study}

- This study validates prior theories about barriers to maternal healthcare for specific target region, highlighting early role of healthcare workers in quality improvement.

- Qualitative design is ideal for capturing variations in themes given the purposes of the study.

- Themes identified are not only highly relevant for the target region and population, but may be helpful for other similar regions.

- Study focuses specifically on healthcare worker perspectives and therefore our analysis focuses on the systems limitations that are directly within the control of these healthcare workers. Further study could include patient-level and communitylevel perspectives of healthcare system barriers affecting pregnant women.

- The small participant pool and the specific geographical focus limit the generalisability of these results.

during deliveries is critical to reduce maternal mortality; however, ensuring that women deliver at facilities with qualified attendants has been a challenge in Ghana. Between 1993 and 2003, while institutional deliveries increased from $85 \%$ to $90 \%$ for the richest quintile, rates for the poorest quintile dropped from $25 \%$ to $19 \%$. Only $45 \%$ of births were attended by a medical practitioner $(79 \%$ in urban areas, $33 \%$ in rural) in 1998. ${ }^{23}$ Over the past decade, the Ministry of Health has made efforts to improve rates of skilled delivery, introducing exemptions from delivery fees in April 2005, ${ }^{3}$ and national insurance covering antenatal care in July 2008. ${ }^{45}$ Despite an increase in utilisation rates increased in some regions, ${ }^{6}$ maternal mortality rates did not improve, ${ }^{7}$ and major challenges remain in providing women ready access to skilled-obstetric services.

The leading direct causes of maternal deaths in Ghana include haemorrhage, infection, eclampsia, obstructed labour and 
Table 1 Range of maternal services provided at the various levels of care

\begin{tabular}{lll}
\hline Health post & Health centre & District hospital \\
\hline Antenatal care & All CHPS Compound services plus & All Health Center services plus \\
Postnatal care & Routine, non-complicated deliveries & management \\
Family planning & & \\
Emergency deliveries only & & \\
(non-complicated) & & \\
\hline
\end{tabular}

septic abortion, most of which occur around the time of labour and delivery. ${ }^{8-10}$ Prevention of management of these complications require care by a skilled-birth attendant and timely access to comprehensive emergency obstetrical care (cEMOC), making referral systems critical for the survival of such women and their babies. $^{11} 12$ There is therefore growing interest in understanding reasons for delayed management of obstetric complications.

According to Thaddeus' and Maine's ${ }^{13}$ '3 delays model', there are three main delays affecting the timely delivery of care to a pregnant women in a facility when complications occur: (I) delays in seeking care, (II) delays in identifying and reaching the appropriate facility and (III) delays in the receiving of quality care once the woman reaches the facility. Several studies have applied this model to local contexts, ${ }^{14-17}$ stressing the importance of eliminating delays occurring before and after the woman in labour arrives at the health facility settings, ${ }^{13} 1516$ and suggesting interventions addressing identified local gaps in various components of the referral process. ${ }^{14} 18^{19}$ Recognising the importance of local provider and consumer inputs to the identification of barriers and ownership of solutions to overcome those barrier, $^{20} 21$ we present a contextual application of Thaddeus and Maine's model in a rural setting in Ghana.

This study aims to understand local healthcare worker (HCW) perspectives on causes of challenges in the referral of obstetric cases in rural Ghana, focusing on type II and III delays, and to identify potential strategies identified by these front-line providers to help address systemic deficits. Including provider perspectives is critical in developing and sustaining change ideas for improvement, a common approach used in quality improvement (QI) initiatives. ${ }^{21} 22$

\section{METHODS}

\section{Project Fives Alive!}

This study was embedded into the Project Fives Alive! (PFA!) project. PFA! is a collaboration between the Institute for Healthcare Improvement and the National Catholic Health Service working in close collaboration with the Ghana Health Service (GHS) to improve maternal and child health outcomes using a QI approach including learning collaboratives and coaching. ${ }^{23}$ By the start of this project in July 2012, the project had reached all health facilities in all 38 districts of the three northernmost regions and all 29 Catholic hospitals in the remaining regions of the country.

\section{Study setting}

Assin North Municipality, located in the Central region of Ghana, has a population of 171499 spread over $1500 \mathrm{~km}^{2}$ and is served by one district hospital, six health centres and four local health posts. In maternal health, health posts typically refer to health centres, which refer to the district hospital. If the district hospital cannot manage any further, it refers ahead to regional and other tertiary hospitals (table 1 ).

Assin North is one of six districts in Ghana selected by PFA! to test strategies to improve existing referral systems to address gaps that contribute to adverse maternal and newborn outcomes. This District was selected due to its relatively high maternal mortality rate (239.2/100 000 live-births, institutional deaths only) and prior experience in QI methodology working with PFA! Lessons

Table 2 Roles of health workers interviewed

\begin{tabular}{ll}
\hline Health cadre (number interviewed) & Obstetric services provided \\
\hline $\begin{array}{l}\text { Community health officer }(\mathrm{CHO})(\mathrm{n}=4) \\
\text { Medical assistant }(\mathrm{MA})(\mathrm{n}=3)\end{array}$ & Antenatal and postnatal care, emergency, non-complicated deliveries only \\
Midwife $(\mathrm{n}=8)$ & $\begin{array}{l}\text { Antenatal and postnatal care, emergency, non-complicated deliveries } \\
\text { Routine, non-complicated deliveries }\end{array}$ \\
Other nurse (hospital emergency and & $\begin{array}{l}\text { First point contact for emergency obstetric and neonatal cases, stabilise and } \\
\text { children's department) }(n=2)\end{array}$ \\
triage cases for advanced management \\
Physician/obstetrician $(n=1)$ & Emergency deliveries requiring advanced management, caesarian sections \\
\hline
\end{tabular}


learned from this 18-month innovation phase focused on maternal mortality will be spread to the rest of the country through the PFA! platform.

\section{Data collection and analysis}

We conducted semistructured interviews with HCWs in four health posts, six health centres and one district hospital in the Assin North Municipality, Ghana (table 2) using interview guides developed based on concepts raised by Thaddeus and Maine, ${ }^{13}$ as well as insights from prior PFA! work in other districts.

This was then piloted with midwives at Maamobi General Hospital in Accra, Ghana prior to the research start date. Feedback was used to adapt the guide to better reflect local context and language.

In collaboration with $P F A$ !, all study participants were recruited on a volunteer basis, and no compensation was given. A month prior to the site visit, invitations were sent to in-charge health staff at all sites, all of whom agreed to participate or delegated to next in-charge staff if they were otherwise indisposed. On the day of the visit to the site, interviews were conducted with these preidentified individuals on the premises.

Eighteen face-to-face semistructured interviews were conducted during a 2-week period in June 2012 representing $51 \%$ of clinicians involved in emergency obstetric delivery in the district. The $49 \%$ who were not interviewed were either not scheduled to work, or were away on vacation at the time of the site visit. Questions were open-ended, and interviews were largely conducted in English (see online supplementary appendix). Respondents were encouraged to use Twi, the local Ghanaian dialect, if they could not find the equivalent words or expressions in English to continue. Interviews were audio-recorded and transcribed verbatim, with any responses in Twi translated to English with expert help prior to analysis. NVivo V.10.0 software was used to analyse the transcripts for common themes regarding barriers and solutions to effective referral and management of obstetric complications. Topics relating to the study aims were identified and coded without predefined categories. After coding was completed, themes were developed and classified, guided by the three-delay framework. A triangulation of data sources, ${ }^{24} 25$ was employed, comparing information from different categories of respondents.

\section{Ethical considerations}

All participants gave informed consent prior to participation in the survey and interviews were conducted in settings where confidentiality could be maintained.

\section{RESULTS}

The study identified a range of challenges of the referral system in Assin North Municipal District, as well as highlighted suggested ideas for improvement. Several major topics emerged related to the referral networks that resulted in type 2 or 3 delays and that had the potential to be directly improved by health centre staff and the health referral network systems: (1) Referral Transport Systems; (2) Communication Barriers; (3) Clinical Skills and Management and (4) Standards of Care and Monitoring.

\section{Referral transport systems}

\section{Accessibility and security}

The closest health facility to the district hospital is $15 \mathrm{~km}$ away. Apart from a stretch of about $100 \mathrm{~km}$ tarred road, all other roads within the district are dirt roads, often in poor condition, which are rendered especially inaccessible during rainy seasons.

Poor road security, especially at night, further compounds the problem of travel. One community health officer (CHO) explained that drivers commuting from remote parts of the district often hesitate to transport patients at night for fear of encountering armed robbers. In some of these communities, no vehicle leaves the village after 18:00.

\section{Reliance on local transportation}

To further complicate the limiting factor of bad roads, the transport capacities of most referring centres are often inadequate. One HCW explains:

\begin{abstract}
Sometimes they hire commercial vehicles and sometimes too they use the motorbike. If there is no commercial vehicle at the station, they will beg someone to use their motorbike to convey them to the nearest health center or hospital, and then maybe somebody's private car. The person might sacrifice.
\end{abstract}

\section{CHO, Health Post}

'Sacrificing' in this regard refers to the liabilities undertaken by the driver or owner of the vehicle in helping to transport a patient. These include physical damages to their vehicle such as transporting a bleeding woman, or loss of revenue to the owner if the commercial vehicle travels to the major town without its full set of passengers.

The National Ambulance service provided an ambulance for the district stationed at the district capital in May 2012, but it was designed for use on tarred rather than dirt roads, making it less ideal for use in many areas in Assin North. It was also underutilised; as of June 2012, this ambulance had responded to only six emergencies, including one obstetric case; this low volume was related to an inability to operate at night due to lack of on-site housing for drivers during night call.

\section{High cost of transportation}

The financial burden to patients of obtaining adequate transportation remains a challenge in Assin North. According to one HCW, there was a recent death in one 
of the nearby villages due to the patient's inability to pay for transportation.

Just this Saturday I heard a lady died in one of the interior villages [...] She was pregnant and not able to deliver. They wanted a car, they contacted the person, the person said he'll charge them 400,000 (Gh cedis, equivalent \$20); [it's] just that they don't have [the money]. So they asked her to go to the TBA. After the baby came the placenta was not coming, and they wasted time, and the lady too... [died].

CHO, Health Post

\section{Inadequate interfacility referral transport equipment}

The use of taxis, lorries and pick-up trucks as referral ambulances between health centres and the district hospital made it difficult to ensure appropriate care while en-route (eg, maintaining intravenous drips and checking vital signs) during the trip. These can take up to several hours from the more remote areas. In 3 of 11 facilities, makeshift ambulances constructed from pick-up trucks originally designed to transport drugs and facility equipment are used to transport patients in critical cases. With such alternative transportation methods, HCWs still face challenges with stabilising and monitoring the patient. As one respondent noted:

[The patient's blood pressure] was falling so we had to put in infusion and in the taxi you know it cannot be hanged...yes, so we needed the ambulance [.... If even there's the need for oxygen it can be given in the ambulance and so on, but in the taxi, [those] kinds of facilities are not [available].

\section{Medical Assistant, Health Center}

\section{Communication barriers \\ Lack of formalised communication systems between key stakeholders}

In Assin North, there are no standard systems for communication, and so any communication is dependent on the initiative of individual providers. Those providers who take the initiative sometimes give their personal phone numbers to patients, call ahead to receiving units or accompany patients. However, this remains challenging because it does not routinely occur in all sites. One HCW described how the lack of advance notice from referring health centres makes it more difficult for hospital staff to prepare adequately:

Apart from that [one] guy (HCW) who calls, the others don't call so you'll be here and such a case comes in. And $[. .$.$] with no...nobody accompanying... it's really a$ challenge. Because if you know [...] somebody is coming with eclampsia... you know you're supposed to prepare first so that you receive [appropriately].

Emergency Nurse, District Hospital
One common limitation is the poor mobile network connectivity especially in the more rural parts of the district. Many providers noted the difficulty in calling ahead to alert receiving units, because of the poor telephone network connectivity in their communities. Lack of feedback from the receiving hospitals is also a noted challenge. Nine of 18 HCWs expressed frustration that they do not receive feedback when they refer patients. From the perspective of receiving hospital staff members, high workload was a frequently reported barrier to providing this feedback.

\section{Poor hand-off management processes}

For effective referrals, HCWs accompanying patients to higher-level facilities should have knowledge of the case or accompanying documentation. However, respondents reported that this is often not the case. For example, a medical assistant at one health centre reported that the nurses who accompany a patient to the receiving hospital are not always able to answer specific questions about what care has already been given.

Other HCWs reported that when they reach the hospital as accompanying health professionals, they sometimes assist in various tasks and procedures, which help facilitate the timely care given to the patient. However, there are other instances where they are mistaken for relatives, and not directly addressed or requested to debrief the cases, especially if they are not in uniform (as is usually the case for late night emergencies). All these factors interfere with an opportunity for communication, limiting the hand-off process and delaying the timely delivery of care to the patient.

\section{Clinical skill limitations}

As shown in table 1, each level of care offers a specific range of services outlined by GHS, and therefore cadres who work at different levels of the system are trained to perform the particular range of services offered at their facilities. However, patients did not always understand this hierarchical structure of capacity and associated limitations. For example, two CHOs explained that clients do not often understand why they need to be referred because they expect the nurse at that facility to be able to help them with all their health needs.

HCWs also reported some challenges related to poor quality within their designated skill set particularly for health workers in the lower levels of care. For instance, one HCW at a receiving hospital described how a patient presented with an advanced condition because the referring facility did not recognise the danger signs on time:

Last time a pregnant woman came here.... And I was saying but there is a doctor at your place, so why did you rush here without a midwife accompanying you, and she said 'Auntie, I had been admitted there for a long time. And each time the doctor came, he said let's wait a bit more, and I was experiencing a lot of discomfort, and I insisted that they discharge me, so they finally reluctantly 
discharged me.' And when she arrived here, true, it was twins. But one was IUFD (macerated) already. So she was able to get the first twin, but the second twin was macerated.

\section{Midwife, District Hospital}

Many HCWs also emphasised the importance of re-training in order to improve their skills and strengthen their skill sets. One midwife in-charge of a health centre noted:

They (staff at the district hospital) need refresher courses... They should allow them to go to workshops so that they will see what is going on.... Me, I always learn from my junior nurses and midwives because $\mathrm{I}$ joined it [midwifery] about 10 years ago, and things are changing. Even the instrument[s] we are using [are] changing.

\section{Midwife, Health Center}

\section{Unreliable standards of care and monitoring \\ Errors in use of existing protocols for referrals}

The basic protocol for making a referral was often the same across all sites: recognise danger signs, stabilise patient, initiate referral, arrange transportation to next appropriate level of care, and complete referral. Even though a National Referral protocol published by the GHS does exist, very few sites adhere to these guidelines. Moreover, the actual process in how it is implemented has notable disparities. For example, even though the national protocol encourages health workers to accompany referred patients, only 5 of 10 respondents reported frequently accompanying the client to the district hospital. Of the remaining five, two accompanied patients in critical cases only and three rarely accompanied the client. Most explained they could not accompany clients because of the low workforce capacity of their sites, even though many understood the importance of accompanying patients to the district hospitals.

\footnotetext{
Somebody who is fitting (or convulsing), a pregnant woman who is fitting... somebody (HCW) needs to accompany. But this is someone who is coming with relatives. They don't know they have to turn the head to the side, [or] the person can aspirate saliva and any other thing $[\mathrm{s}]$.
}

\section{Nurse, District Hospital}

\section{Poor documentation and monitoring of indications for referral}

Another referral challenge is the inadequate documentation of referrals. Five sites regularly used recommended GHS referral forms, one documented referrals in clients' antenatal record cards, while the others used prescription forms and other paper forms. The information documented on these sheets was often inadequate. Of all 11 facilities sampled, only six maintained a register on site in which to document referred cases.
Indications for referral were recorded only in hospital records but included limited depth and context.

\section{Health worker perceptions of patient-level barriers}

Although beyond the main goals of the study, HCWs also described their perceptions of the role of patientlevel barriers to appropriate referrals. These included prohibitive financial costs, sociocultural beliefs related to fear of blood transfusions and of death in bigger facilities and past negative experiences such as delays at hospitals, or inconsiderate treatment by hospital staff.

\section{PRELIMINARY IDEAS FOR IMPROVEMENT}

On the basis of referral challenges highlighted above, HCWs highlighted the following as potential strategies to the referral challenges in Assin North: standardising implementation of the referral protocol, improving transportation systems, ensuring reliable data reporting and management systems, actively engaging the community and providing continuous training for health staff.

\section{DISCUSSION}

Using provider narratives, we identified important gaps in referral processes due to root causes in four domains: (1) transportation, (2) communication, (3) clinical skills and management and (4) standards of care and monitoring. These findings concur with some of the major barriers and challenges identified in previous work in similar settings, ${ }^{26}$ as well as a number of context-specific ideas for improving access to obstetric care in Ghana. Although these four areas describe elements of delays II and III in Thaddeus and Maine's model, they still present real challenges facing a pregnant woman in Assin North, even if she is able to overcome delay I.

Similar to our findings, transportation has been noted as one of the most documented barriers to timely referral of pregnant women in resource-poor settings, ${ }^{19}$ especially in more remote rural regions. Poor road networks and insufficient transportation options coupled with high transport costs have confronted the referral system in Assin North. The Ghana National Ambulance system was introduced in 2004 to help alleviate the growing demand for emergency transport services, but its supply has not matched the current need. A recent study showed that during 2006-2007 the highest number of ambulance use nationally were made for delivery complications, ${ }^{27}$ further demonstrating the need for more affordable and effective options for emergency obstetric transport. Rural communities in other parts of the world with little access to ambulance services have developed innovative methods to address the transportation challenge, including community financing schemes in the form of emergency fuel funds, ${ }^{28} 29$ and autorickshaws. ${ }^{30}$ These could be replicated in Assin North, in addition to ongoing plans to scale-up current provisions between the GHS and certain local transport unions allowing drivers to transport pregnant women without charge, in 
return receiving coupons redeemable for monthly prises.

In care delivery at the district level, there is a need for formalised systems of communication to ensure effective coordination of care. In Assin North, poor mobile network connectivity coupled with a lack of standard systems for follow-up and feedback weakens the referral process. Several studies have underscored the critical need for communication for maternal health, ${ }^{31-33}$ with use of low-cost radio systems, ${ }^{34}$ and four-wheeled referral vehicles, ${ }^{35}$ helping achieve lower maternal mortality rates in similar rural communities. Assin North could replicate such efforts and encourage other innovative approaches to using modern technologies that improve communication between the various stakeholders in care delivery.

A hospital-based study in Nigeria found that referrals to the hospital for appropriate management were made only after prolonged delay and onset of complication, and health centres often misdiagnosed cases. ${ }^{36}$ Likewise, in Assin North, deficits were noted in recognising danger signs, stabilising patients, and handing over to receiving staff. These represent fundamental inadequacies in training and retraining that warrant further investigations into ways to eliminate such direct threats to patient safety. Further compounding these inadequate skillsets are marked inequalities existing in the standards of care provided. These were noted in the use of accompanying nurses, calling ahead to alert receiving units, following up with patients, and documenting cases. Moore in 1994 proposed a safe motherhood checklist which aims to increase awareness of safe motherhood concepts and practices to both providers and community members as a means to reduce maternal deaths, especially in resource poor settings. ${ }^{37}$ Regions like Assin North might benefit from such an intervention, especially as it addresses identified problems related to both clinical skills limitations and unreliable standards of care.

\section{Developing comprehensive solutions to obstetric referral challenges: the value of HCWs}

The Institute for Healthcare Breakthrough Series proposes a collaborative model for accelerating QI that places emphasis on the important role of healthcare providers in stimulating and sustaining change. ${ }^{22}$ This inspired the design of our study in the way we generated primary data from provider narratives, and stimulated further discussions about most effective strategies for designing interventions. Figure 1 is a process map developed with help of providers that stresses the critical role of HCWs in supplying such practical information (compared to patients, other key stakeholders). Second, it illustrates how the different barriers to care are intricately linked, arguing for solutions to referral challenges that take on a more holistic approach. For example if an ambulance is introduced to transport pregnant women to the nearest facility (addressing transportation barrier) but no interventions are put in place to promptly and accurately triage (clinical skills limitation), bottlenecks would still exist in the referral process, and health outcomes may not necessarily improve. Recognising that providers may more reliably influence phases II and III barriers, we argue that phase I could also remotely be influenced if interventions modulate the experience patients have with the healthcare system in general.

An important next step would be to determine how to best target interventions that address the identified barriers to referrals for pregnant women in Assin North and measure their impact within the context of the larger project. The Lancet Neonatal Survival Series called for action to addressing neonatal mortality using both facility and community-based interventions, highlighting the important role of maternal health outcomes in achieving improvement. ${ }^{38} 39$ Such multipronged approach could be implemented in Assin North, with the added advantage from this study of targeting identified underlying barriers to care, a theme mentioned but not expanded in the Lancet series. QI involving HCWs presents another framework for using ideas from the grassroots to effecting change on local levels. Assin North has begun this process, and further work would be needed to assess utility, effectiveness and outcomes as they pilot interventions to reduce maternal mortality in the district.

\section{Study limitations}

The small participant pool and the specific geographical focus limit the generalisability of these results. However, this study has identified themes that are highly relevant for the target region and population, with a good chance that recommendations may hold for other regions with similar demographic and provisional characteristics.

Additionally, this study focused specifically on HCW perspectives and therefore our analysis focuses on the system limitations that are directly within the control of these HCWs. Further study is needed to fully understand patient-level perspectives of healthcare system barriers, as well as community-level and individual-level factors that also influence how and when women access critical EMOC services.

\section{CONCLUSIONS}

A significant component of maternal deaths in the developing world are attributable to referral systems that are confronted with multiple barriers in the care delivery process, prompting the need to investigate highimpact bundle approaches that target these bottlenecks concurrently. HCWs play a key role in this process, and are currently engaged in Assin North through PFA! to further define the challenges highlighted in this paper, with plans to test and retest change ideas according to the improvement model. Still, more work remains to be carried out to refine and evaluate these interventions as 
Referring Unit Protocol

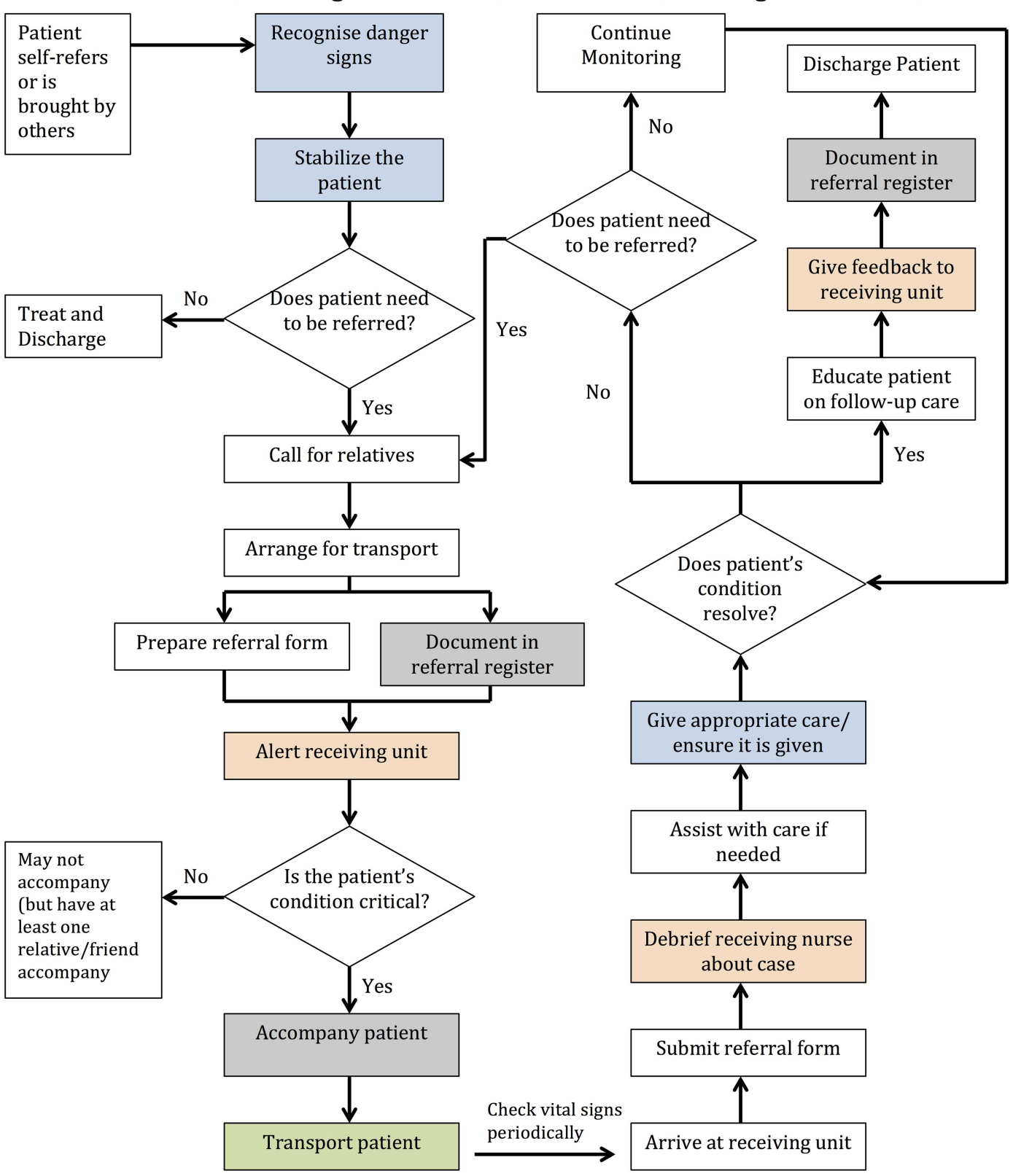

Figure 1 An ideal baseline referral process map, showing major gaps (coloured boxes) in referral processes reported for Assin-North Municipality. (Gaps colour-coded to reflect major domains of referral challenges. Green=transportation, orange=communication; blue=clinical skills; grey=standards of care.)

they apply to Assin North Municipality, with implications that could potentially inform similar work in other settings that strive to improve maternal outcomes in the face of limited resources.

\section{Author affiliations}

${ }^{1}$ Harvard Medical School, Boston, Massachusetts, USA

${ }^{2}$ Partners in Health, Boston, Massachusetts, USA

${ }^{3}$ Institute for Healthcare Improvement, Boston, Massachusetts, USA

${ }^{4}$ Project Fives Alive!/Institute for Healthcare Improvement, Accra, Ghana

Acknowledgements The authors would like to thank staff of NCHS/Project Fives Alive! in Accra, Ghana who helped coordinate the site visit and helped give feedback on preliminary results of the study. They also thank the Assin North District Health Directorate and the St Francis Xavier Hospital at Assin
North who were instrumental in shaping design of data collection and coordinating logistics while in the district. The authors specially thank $\mathrm{Dr}$ Ernest Asiedu of PFA! for spearheading all logistical details during the site visit.

Contributors HA, LRH and SS-T conceived the study. HA undertook interviews and analysis of the data. LRH, AM and PB helped with editing the content of the paper. All authors contributed to the final version of the manuscript.

Funding This study was completed with funding by the Harvard Medical School Scholars in Medicine Office (HA).

\section{Competing interests None.}

Ethics approval The protocol was reviewed and approved by the Harvard University Faculty of Medicine Committee on Human Studies and the Ghana Health Service Ethical Review Committee. 
Provenance and peer review Not commissioned; externally peer reviewed.

Data sharing statement Interview transcripts outlined in the semistructured questionnaires (see online supplementary appendix 1) have been formally coded and analysed into themes according to the described methodology in the current submission.

Open Access This is an Open Access article distributed in accordance with the Creative Commons Attribution Non Commercial (CC BY-NC 3.0) license, which permits others to distribute, remix, adapt, build upon this work noncommercially, and license their derivative works on different terms, provided the original work is properly cited and the use is non-commercial. See: http:// creativecommons.org/licenses/by-nc/3.0/

\section{REFERENCES}

1. Bhutta ZA, Chopra M, Axelson $\mathrm{H}$, et al. Countdown to 2015 decade report (2000-10): taking stock of maternal, newborn, and child survival. Lancet 2010;375:2032-44.

2. Hussein J, Hundley V, Bell J, et al. How do women identify health professionals at birth in Ghana? Midwifery 2005;21:36-43.

3. Witter S, Arhinful DK, Kusi A, et al. The experience of Ghana in implementing a user fee exemption policy to provide free delivery care. Reprod Health Matters Elsevier, 2007;15:61-71. http://www. rhm-elsevier.com/article/S0968-8080(07)30325-X/abstract

4. Gobah FK, Zhang L. The National Health Insurance Scheme in Ghana: prospects and challenges: a cross-sectional evidence. Glob $J$ Health Sci 2011;3:90-101.

5. HERA and Health Partners Ghana. Evaluation of the free maternal health care initiative in Ghana. UNICEF Final Report.

6. Penfold S, Harrison E, Bell J, et al. Evaluation of the delivery fee exemption policy in Ghana: population estimates of changes in delivery service utilization in two regions. Ghana Med $J$ Ghana Medical Association; 2007;41:100-9. http://www.pubmedcentral.nih gov/articlerender.fcgi? artid=2279083\&tool=pmcentrez\&rendertype=abstract

7. Bosu WK, Bell JS, Armar-Klemesu M, et al. Effect of delivery care user fee exemption policy on institutional maternal deaths in the Central and Volta regions of Ghana. Ghana Med J Ghana Medical Association; 2007;41:118-24. http://hdl.handle.net/2164/224

8. Khan KS, Wojdyla D, Say L, et al. WHO analysis of causes of maternal death: a systematic review. Lancet 2006;367:1066-74. http://www.ncbi.nlm.nih.gov/pubmed/16581405

9. Lee QY, Odoi AT, Opare-Addo H, et al. Maternal mortality in Ghana: a hospital-based review. Acta Obstet Gynecol Scand 2012;91:87-92. http://www.ncbi.nlm.nih.gov/pubmed/21793813

10. Geelhoed DW, Visser LE, Asare K, et al. Trends in maternal mortality: a 13-year hospital-based study in rural Ghana. Eur J Obstet Gynecol Reprod Biol Elsevier Ltd; 2003;107:135-9. http:// www ncbi.nlm nih gov/pubmed/12648857

11. Ronsmans C, Etard JF, Walraven G, et al. Maternal mortality and access to obstetric services in West Africa. Trop Med Int Health Blackwell Science Ltd; 2003;8:940-8. http://www.ncbi.nlm.nih.gov/ pubmed/14516306

12. Health F. Situation analyses of emergency obstetric care: examples from eleven operations research projects in West Africa. The Prevention of Maternal Mortality Network. Soc Sci Med 1995;40:657-67.http://www.ncbi.nlm.nih gov/pubmed/7747201

13. Thaddeus $S$, Maine $D$. Too far to walk: maternal mortality in context Soc Sci Med Elsevier, 1994;38:1091-110. http://www.ncbi.nlm.nih. gov/pubmed/8042057

14. Alisjahbana $A$, Williams $C$, Dharmayanti $R$, et al. An integrated village maternity service to improve referral patterns in a rural area in West-Java. Int J Gynaecol Obstet Off Organ Int Fed Gynaecol Obstet Elsevier : Limerick, 1995;48(Suppl):S83-94. http://search.ebscohost.com/login. aspx?direct=true \&db=cmedm\&AN=7672178\&site=ehost-live \&scope=site

15. Ozumba BC, Nwogu-lkojo EE. Avoidable maternal mortality in Enugu, Nigeria. Public Health 2008;122:354-60. http://www.ncbi. nlm.nih.gov/pubmed/17959207

16. Barnes-Josiah D, Myntti C, Augustin a. The "three delays" as a framework for examining maternal mortality in Haiti. Soc Sci Med 1998;46:981-93. http://www.ncbi.nlm.nih.gov/pubmed/9579750

17. Griffiths P, Stephenson R. Understanding users' perspectives of barriers to maternal health care use in Maharashtra, India. J Biosoc Sci 2001;33:339-59.

18. Hofman JJ, Dzimadzi C, Lungu K, et al. Motorcycle ambulances for referral of obstetric emergencies in rural Malawi: do they reduce delay and what do they cost? Int J Gynaecol Obstet Off Organ Int
Fed Gynaecol Obstet 2008;102:191-7. http://www.ncbi.nIm.nih.gov/ pubmed/18555998

19. Hussein J, Kanguru L, Astin M, et al. The effectiveness of emergency obstetric referral interventions in developing country settings: a systematic review. PLoS Med 2012;9:e1001264. http://www. pubmedcentral.nih.gov/articlerender.fcgi? artid $=3393680 \&$ tool=pmcentrez\&rendertype $=$ abstract

20. Berwick DM. The science of improvement. JAMA 2008;299:1182-4.

21. Wilson T, Berwick DM, Cleary PD. What do collaborative improvement projects do? Experience from seven countries. Jt Comm J Qual Saf 2003;29:85-93. http://www.ncbi.nlm.nih.gov/ pubmed/21312329

22. Kilo CM. A framework for collaborative improvement: lessons from the Institute for Healthcare Improvement's Breakthrough Series. Qual Manag Health Care ASPEN PUBLISHERS, INC.; 1998;6:1-13. http://journals.Iww.com/qmhcjournal/Fulltext/1998/06040/A Framework_for_Collaborative_Improvement_Lessons.1.aspx

23. Twum-Danso NAY, Akanlu GB, Osafo $\mathrm{E}$, et al. A nationwide quality improvement project to accelerate Ghana's progress toward Millennium Development Goal Four: design and implementation progress. Int J Qual Heal Care J Int Soc Qual Heal Care ISQua 2012;24:601-11. http://www.ncbi.nlm.nih.gov/pubmed/23118097

24. Ritchie J, Lewis J. Qualitative research practice: a guide for social science students and researchers [Internet]. Ritchie J, Lewis J. eds. Quality research. SAGE, 2003:356.http://books.google.co.uk/books? id=e6EO83ZKGYoC

25. Green J, Thorogood N. Qualitative methods for health research [Internet]. In: Silverman D, ed. Introduction to quality methods. Sage Publications, 2004:262. http://prism.talis.com/greenwich-ac/items/ 587256

26. Gabrysch S, Campbell OMR. Still too far to walk: literature review of the determinants of delivery service use. BMC Pregnancy Childbirth 2009;9:34

27. Nguyen KT. Prehospital care in Ghana: Utilization of the national ambulance service [Internet]. Acad Emerg Med, Blackwell Publishing Ltd, 2011:S243-4. http://ovidsp.ovid.com/ovidweb.cgi? $\mathrm{T}=\mathrm{JS} \& P A G E=$ reference\&D=emed10\&NEWS=N\&AN=70473920

28. Shehu D, Ikeh AT, Kuna MJ. Mobilizing transport for obstetric emergencies in northwestern Nigeria. The Sokoto PMM Team. Int J Gynaecol Obstet Off Organ Int Fed Gynaecol Obstet 1997;59(Suppl 2):S173-80. http://ovidsp.ovid.com/ovidweb.cgi?T=JS\&CSC=Y\& NEWS $=$ N\&PAGE $=$ fulltext $\& D=$ med $4 \& A N=9389629$

29. Macintyre K, Hotchkiss DR. Referral revisited: community financing schemes and emergency transport in rural Africa. Soc Sci Med 1999;49:1473-87. http://ovidsp.ovid.com/ovidweb.cgi? $\mathrm{T}=\mathrm{JS} \& P A G E=$ reference\&D=emed4\&NEWS=N\&AN=1999399204

30. Paul B, Mohapatra B, Kar K. Maternal deaths in a tertiary health care centre of Odisha: an in-depth study supplemented by Verbal Autopsy. Indian J Community Med 2011;36:213-16. http://www.ncbi. nlm.nih.gov/entrez/query.fcgi? $\mathrm{cmd}=$ Retrieve \&db=PubMed\&dopt=Citation\&list_uids $=22090676$

31. Braa K, Purkayastha S. Sustainable mobile information infrastructures in low resource settings. Stud Health Technol Inform 2010;157:127-32. http://www.ncbi.nlm.nih.gov/pubmed/20543378

32. Kuntiya K, Mavunduse D. Improving home based care through mobile phones in Malawi. First International Conference M4D Mobile Communication Technology Development. 2008.

33. Noordam AC, Kuepper BM, Stekelenburg J, et al. Improvement of maternal health services through the use of mobile phones. Trop Med Int Health Blackwell Publishing Ltd; 2011;16:622-6. http://www. ncbi.nlm.nih.gov/pubmed/21342374

34. Fournier P, Dumont A, Tourigny C, et al. Improved access to comprehensive emergency obstetric care and its effect on institutional maternal mortality in rural Mali. Bull World Health Organ 2009;87:30-8.

35. Samai O, Sengeh P. Facilitating emergency obstetric care through transportation and communication, Bo, Sierra Leone. The Bo PMM Team. Int J Gynaecol Obstet Off Organ Int Fed Gynaecol Obstet 1997;59(Suppl 2):S157-64. http://www.ncbi.nlm.nih.gov/pubmed/ 9389627

36. Onwudiegwu U, Ezechi OC. Emergency obstetric admissions: late referrals, misdiagnoses and consequences. J Obstet Gynaecol 2001;21:570-5. http://www.ncbi.nlm.nih.gov/pubmed/12521770

37. Moore M. Promoting safer motherhood: a checklist for communications and training. Mothers Child 1994;13:6-7.

38. Lawn JE, Cousens S, Zupan J. Neonatal Survival 14 million neonatal deaths: when? Where? Why? Lancet 2005;365:891-900.

39. Darmstadt GL, Bhutta ZA, Cousens S, et al. Neonatal Survival 2 Evidence-based, cost-effective interventions: how many newborn babies can we save? Lancet 2005;365(panel 1):977-88. 